\title{
Role of osteopontin in decidualization and pregnancy success
}

\author{
Xiao-Bo Wang ${ }^{1, *}$, Qian-Rong Qi ${ }^{2, *}$, Kai-Lin Wu and Qing-Zhen Xie ${ }^{2}$ \\ ${ }^{1}$ Medical College of Wuhan University, Wuhan, People's Republic of China and ${ }^{2}$ Center for Reproductive Medicine, \\ Renmin Hospital of Wuhan University, Wuhan, People's Republic of China
}

Correspondence should be addressed to Q-Z Xie; Email: qingzhenxie@hotmail.com

*(X-B Wang and Q-R Qi contributed equally to this work)

Reproduction (2020) 159 X3

The authors apologise for errors in the affiliations listed in their article published in the May 2018 issue of this journal (Vol 155 Iss 5 pages 423-432). The corrected author list and affiliations are published below:

Xiao-Bo Wang ${ }^{1, *}$, Qian-Rong Qi ${ }^{1, *}$, Kai-Lin Wu² and Qing-Zhen Xie ${ }^{1}$

${ }^{1}$ Center for Reproductive Medicine, Renmin Hospital of Wuhan University, Wuhan, People's Republic of China and

${ }^{2}$ Medical College of Wuhan University, Wuhan, People's Republic of China 